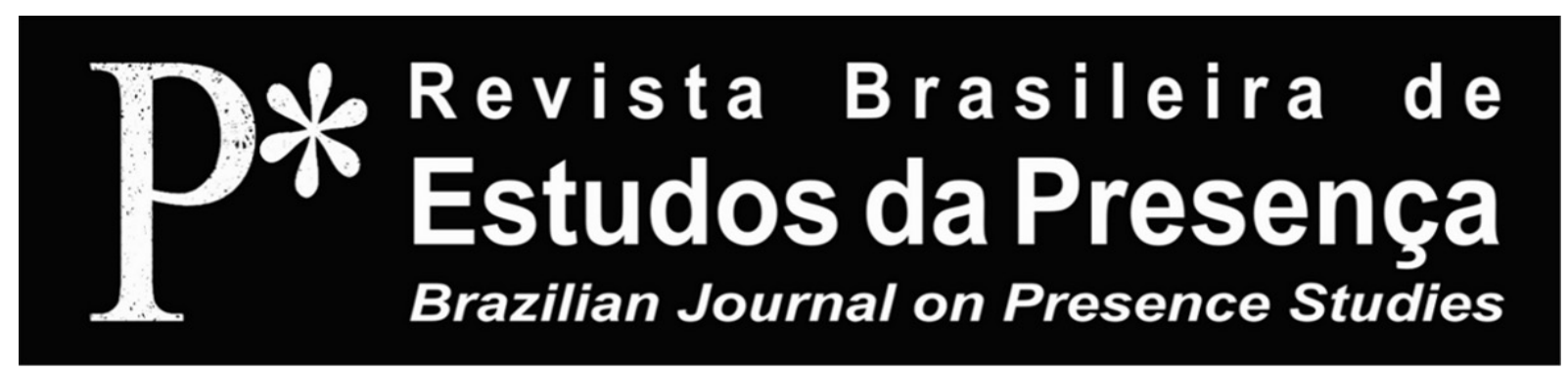

E-ISSN 2237-2660

\title{
La Théâtralité, entre Théories et Pratiques: un regard sur la démarche du Théâtre du Soleil
}

\author{
Suzana Thomaz \\ Université Paris 8 - Paris, France
}

RÉSUMÉ - La Théâtralité, entre Théories et Pratiques: un regard sur la démarche du Théâtre du Soleil - Cet article aborde la problématique actuelle de la notion de théâtralité, couvrant ses nombreuses définitions, critiques et approches sur la pratique théâtrale en Occident, d'après plusieurs théoriciens et artistes depuis le début du $\mathrm{XX}^{\mathrm{e}}$ siècle. Appuyé par une approche historique de la théâtralité, l'article revisite notamment ses rapports avec la performance et la performativité, donnant ainsi des fondements pour analyser l'émergence de la théâtralité dans la création de Macbeth (2014) au Théâtre du Soleil.

Mots-clés: Théâtralité. Performativité. Théâtre du Soleil. Ariane Mnouchkine. Processus Créatif.

ABSTRACT - Theatricality, between Theories and Practices: a glance at Théâtre du Soleil's approach - The present article approaches the contemporary problematics around the notion of theatricality; its various definitions, criticisms and approaches concerning performing arts in the West, according to theorists and artists since the beginning of the $20^{\text {th }}$ century. Supported by a historical approach of theatricality, this article revisits its relation to the notions of performance and performativity, providing material to analyze the emergence of theatricality in the creation of Macbeth (2014) at the Théâtre du Soleil. Keywords: Theatricality. Performativity. Théâtre du Soleil. Ariane Mnouchkine. Creative Process.

RESUMO - Teatralidade, entre Teorias e Práticas: um olhar sobre a abordagem do Théâtre du Soleil - Este artigo aborda a problemática atual da noçáo de teatralidade, tratando de suas diversas definiçóes, críticas e abordagens no que se refere à prática teatral ocidental, de acordo com teóricos e artistas desde o início do século XX. Baseado em uma abordagem histórica da teatralidade, o artigo revisita sobretudo suas relaçóes com as noçóes de performance e de performatividade, criando fundamentos para analisar a emergência de teatralidade na criação de Macbeth (2014), no Théâtre du Soleil.

Palavras-chave: Teatralidade. Performatividade. Théâtre du Soleil. Ariane Mnouchkine. Processo Criativo. 
Un concept souvent connoté et critiqué au-delà du milieu théâtral, la notion de théâtralité pose problème parmi artistes et théoriciens depuis ses premiers emplois au début du XX $\mathrm{XX}^{\mathrm{e}}$ siècle. À partir des années 1980, pourtant, le terme se remet à l'ordre du jour, grâce à l'irruption de discussions autour des notions contemporaines de transdisciplinarité et de performativité, entre autres. Mais de quelle théâtralité parle-t-on? Comment la produire, ou la rendre possible ? Pourquoi ce terme engendre-t-il autant de connotations dépréciatives? Y aurait-il du théâtre sans théâtralité?

Dans son dictionnaire du théâtre, Patrice Pavis (2003, p. 358) souligne la difficulté de définir ce concept: "Notre époque théâtrale se caractérise par la recherche de cette théâtralité trop longtemps occultée. Mais le concept a quelque chose de mythique, de trop général, voire d'idéaliste et d'ethnocentriste". Dans la préface d'une revue spécialisée abordant le sujet de la théâtralité et publiée en 2002, Josette Féral (2002, p. 4) affirme: "C’est précisément parce que la notion de théâtre a changé, que nous devons constamment redéfinir la notion de théâtralité".

Nous nous penchons dans ces questionnements sur la théâtralité autour des créations du Théâtre du Soleil - et plus précisément autour de la création de Macbeth, à laquelle nous avons participé, entre 2013 et 2015. Introduisons donc notre sujet sur une brève définition proposée par Josette Féral (2012, p. 9):

[la théâtralité serait] un processus selon lequel le sujet (ici l'artiste) modifie la perspective sur les choses afin d'obliger le spectateur à 'les voir de façon différente'. [...] Elle implique l'existence d'un décalage entre la vie et la scène, entre l'action naturelle et l'action théâtralisée.

Dans toute création du Théâtre du Soleil, chaque élément présent sur scène (et pour la scène) sert à soutenir le jeu, et à faire en sorte qu'il soit transposé et conventionné. Tout est créé avec une notion d'ensemble, au fur et à mesure que les répétitions avancent et que le spectacle prend forme. Cette théâtralité dépend, entre autres, des conventions créées avec les bons outils de l'espace, des costumes, de la musique et de l'éclairage, pour soutenir le jeu des acteurs. Pourtant, afin de rendre possible ce "décalage entre la vie et la scène" dont parle Féral, elle dépend aussi bien de la performance des artistes que de la reconnaissance et du regard du spectateur. 
Un événement considéré comme théâtral aujourd'hui ne l'aurait pas forcément été (ni ne le sera) dans une autre époque, puisque nous admettons, dans une conception contemporaine du terme, que c'est le regard du spectateur qui crée l'émergence de la théâtralité. C'est un phénomène qui dépend à la fois d'un sujet/objet regardé (le performeur ou la scène) émetteur/porteur de signes, et d'un autre regardant (le spectateur) capable d'identifier et de déchiffrer ces signes en tant que tels ${ }^{1}$. Cette formule peut varier en fonction d'innombrables conditions, dont le bagage et milieu culturel, l'époque et le lieu de représentation, le contexte social et politique en question, le contact entre les deux, et, bien entendu, du désir de communiquer (transmettre et recevoir) au travers de conventions, signes et codes communs.

Malgré sa racine ancienne, attribuée au latin classique theatralis, l'implication du terme dans les théories des arts du spectacle n'est parue qu'au début du XX $\mathrm{X}^{\mathrm{e}}$ siècle - dans les publications de Nicolas Evreinov sur la conception de teatralnost. Malgré la reprise du terme dans les années 1950 par Roland Barthes, son intérêt ne fut relevé que dans les années 1980, remis en cause par la grande vague de transdisciplinarité des arts et par la performance. Depuis lors, la théâtralité fait l'objet de différentes interprétations, portant sur des domaines et consignes distinctes, qui ne sont pas toujours propres au théâtre. Dans l'ère du (post) postdramatique et du (post) postmoderne, où les théoriciens s'interrogent sur l'épuisement de la transgression des frontières artistiques, revisiter la notion de théâtralité reste à l'ordre du jour.

\section{La Performance entre en Jeu}

Le dernier grand bousculement des repères liés à la notion de théâtralité dans le domaine artistique découle des discussions et mouvements déclenchés dans les années 1970 autour de la performance (et donc de la performativité) qui, à la fois, s'insère dans le domaine théâtral et le rejette - tout en rejetant, ainsi, la notion de théâtralité. D'une part, les performance studies, menées par Richard Schechner, ont privilégié les sphères sociales de la performativité et de l'acte artistique de "performer", et ont relativisé le rôle de l'artiste (performeur) et du spectateur. De l'autre, avec la notion de Performance Art, l'irruption de la performance dans les manifestations et études 
théâtrales explose progressivement les limites du (re)présentable et du scénique. L'effet de présence, le caractère fictionnel, et la vérité du corps théâtral sont constamment remis en question par artistes et théoriciens.

Comme l'analyse Janelle Reinelt (2012, p. 13), “[...] cet usage du terme 'performance' appartient à l'histoire du théâtre d'avant-garde, ou anti-théâtre, et est fondé sur le rejet des aspects de la pratique théâtrale traditionnelle". Il ne faut pourtant pas oublier que, malgré leur racine, les termes "performance" et "performativité" ne sont pas interchangeables! En dehors de ce repère historique, Josette Féral (2012, p. 11) nous rappelle:

La théâtralité a aussi à voir avec le corps, les pulsions, le désir et donc avec la performativité. [...] La performativité est ce qui permet de lire en toute représentation une activité unique et en perpétuel mouvement chaque fois qu'elle a lieu. La théâtralité, par ailleurs, est ce qui permet au spectateur de repérer dans ce mouvement performatif quelques stases et donc d'inscrire la scène dans l'acte de représentation.

Citée comme une référence sur ce sujet par de nombreux théoriciens - tels qu'André Helbo, Marlvin Carlson, Patrice Pavis, et Richard Schechner - Josette Féral assume, d'une part, le sage rôle de théoriser la pratique actuelle, et d'autre part, de revisiter les théories les plus pertinentes du $\mathrm{XX}^{\mathrm{e}}$ siècle, les actualisant et les remettant en question. Son approche met la réception du spectateur en évidence dans l'émergence de la théâtralité, et ouvre le dialogue entre performativité et théâtralité, perspectives qui nous semblent fondamentales pour traiter de la question de la théâtralité au Théâtre du Soleil.

L'approche de Féral nous semble aussi particulièrement intéressante pour introduire les notions des clivages et de l'altérité, qui sont à la base de sa théorie de la théâtralité - et précisément où cette notion rencontre celle du spectaculaire : elle met au centre la reconnaissance de certains repères théâtraux et d'un espace autre que celui du quotidien, avec des lois et des règles spécifiques qui demandent au spectateur un regard différent, un regard autre. Ce regard est toujours double, dû à la dualité fiction/réalité, propre au spectaculaire, où se place la dénégation du spectateur - le fameux "je sais, mais quand même..." ${ }^{2}$. Cette approche propose aussi que l'émergence de la théâtralité soit comprise comme un processus non-autonome et 
non-qualitatif : c'est-à-dire qu'elle ne se produit pas sans le processus constamment renouvelé regardé-regardant, et ne se mesure pas en intensité ni en degré.

Avec la notion glissante de théâtralité mieux encadrée, nous suivrons la discussion en couvrant d'autres approches théoriques sur la théâtralité, avant de passer, dans un deuxième temps, à l'étude de cas du Théâtre du Soleil.

\section{Théâtralité, Connotations et Critiques}

La notion de théâtralité contourne régulièrement les sciences littéraires, sociales, la philosophie, la psychologie et l'anthropologie. Avant d'arriver aux théories les plus récentes, où son occurrence devient moins mystifiée, nous passerons par quelques contradictions et critiques subies par ce terme depuis le début du $\mathrm{XX}^{\mathrm{e}}$ siècle, afin de contextualiser historiquement son parcours, ainsi que les théories que nous aborderons par la suite. Dans l'essai Résistance à la théâtralité (2012), Marvin Carlson relève plusieurs problématiques entourant l'usage du terme de théâtralité.

Nous en remarquons deux, qui seront abordées plus attentivement dans cet article: 1) la résistance à sa légitimation, renforcée par la prédominance du réalisme et du textocentrisme dans le théâtre occidental traditionnel; 2) son rapport contradictoire et ambigu aux concepts de performance et de performativité.

Entre l'une et l'autre, nous pourrons mieux encadrer et relativiser la question de la théâtralité telle que nous la retrouvons actuellement au Théâtre du Soleil.

Nous introduisons ces problématiques au travers de deux anecdotes, qui illustrent ensemble l'enjeu d'acceptation de la théâtralité à travers le dernier siècle, tant par opposition au réalisme occidental, que par rejet du non-performatif.

\section{Un Siècle entre Moscou et Paris}

En novembre 1906 à Moscou, Meyerhold met en scène la pièce Baraque de foire, du poète Alexandre Blok. Ce poète lyrique, comme beaucoup d'autres artistes de l'époque, voit la théâtralité au théâtre comme un manque délibéré de sincérité, de vérité et de lyrisme; et donc la représentation du grotesque, du schématique, du stéréotypé. 
D'après Gérard Abensour (1982, p. 671), théoricien qui a analysé le journal et les notes publiées de Blok, "dans cette optique, être théâtral, c'est être faux”. Le poète n'apprécie pas cette théâtralité, et ressent en Meyerhold l'homme moderniste, de veine expressionniste, dont le théâtre manque de psychologie - caractère de ce qui apporte, pour lui, la nourriture à l'âme. Blok va donc utiliser un dispositif dramaturgique pour essayer de créer une distance entre théâtre et théâtralité, afin de dénoncer sa fausseté et retrouver la sincérité qu'il recherche dans des personnages humanisés. Abensour (1982, p. 672) décrit la situation telle qu'elle s'est passée en 1906, et critique la démarche de Blok:

Le poète introduit dans sa pièce un personnage dont, au premier abord, rien ne suggère la théâtralité. Il s'agit de l'Auteur. Celui-ci apparaît sur la scène en costume de ville, il proteste avec la plus grande sincérité de ses bonnes intentions auprès du public. [...] Or, par un effet inversé, voilà que ce personnage antithéâtral révèle sa théâtralité intérieure, c'est-à-dire son absence de vérité et d'humanité. L'intrigue qu'il avait imaginée n'était qu'un cliché théâtral usé jusqu'à la corde, dépourvu de toute vérité profonde. [...] Tout l'être de Blok s'insurge contre une telle pauvreté de conception, contre une vulgarité qui est ici synonyme de théâtralité (c'est nous qui soulignons).

Dans l'article de Gérard Abensour, publié en 1982, nous pouvons déjà repérer une certaine remise en question de cette conception de théâtralité représentant tout ce qui relève du "mauvais goût", ou qui échappe au "réalisme sain" ${ }^{3}$ tant voulu par le théâtre en vogue du début du siècle.

Cent sept automnes après l'histoire de Blok, nous retrouvons la situation suivante, retirée de nos notes personnelles pendant le processus de création de Macbeth au Théâtre du Soleil:

Le 5 novembre 2013 : Maurice et Juliana improvisent une scène du deuxième acte, où le couple Macbeth se rejoint en cachette après l'assassinat du roi Duncan. Ariane interrompt l'improvisation avant la fin de la scène et la commente ainsi : 'Ce n'est pas ça. Vous étiez dans la théâtralité et non pas dans le théâtre...' Cela m'a abasourdie. Pourquoi avait-elle utilisé ce terme ?! Puis elle nous interpelle, nous qui regardons la scène depuis les gradins : 'Mais vous comprenez ce que je veux dire quand je parle de théatralité, non?'. Elle continue à commenter l'improvisation, en utilisant des mots comme jeu trop poussé, état superficiel, clichés, stéréotypes et manque de vérité. Elle a évidemment senti le be- 
soin de préciser sa pensée, la théâtralité étant un terme très connoté. Une fois cette connotation péjorative insérée dans un contexte artistique, il devient nécessaire de lever toute ambigüité .

Mnouchkine utilisa à plusieurs reprises le mot de théâtralité avec cette même connotation péjorative au cours du processus créatif, ce qu'elle n'aurait peut-être pas fait dans un contexte différent. Il ne s'agit pas ici de porter un avis sur l'usage de cette expression par la metteuse en scène, dans l'intimité des répétitions. Il nous a semblé intéressant d'observer qu'elle emploie la notion de théâtralité dans un sens négatif, l'adjectif "théâtral" exprimant quant à lui le sens de sa quête artistique, ce qu'elle recherche profondément : les moments de vérité du Théâtre, avec un grand T.

\section{Le Théâtral et le Réalisme}

Dans le siècle qui sépare Mnouchkine de Blok, nous observons un grand paradoxe: si le rapport et la quête du réalisme ont tout bousculé, la connotation péjorative de théâtralité persiste encore, en opposition à l'authenticité (la vérité) de la performance théâtrale. Pour introduire le premier sujet, citons Marvin Carlson (2012, p. 31-32):

Le déclin de l'intérêt porté au terme 'théâtralité' [...] a sans aucun doute renforcé la prédominance du réalisme dans le théâtre occidental conventionnel du vingtième siècle. Du point de vue de la tradition réaliste, la théâtralité est également perçue de manière très négative, puisque celleci, lorsqu'elle apparaît ou lorsqu'elle est reconnue comme telle, met en question l'illusion qui s'évertue à nier, tout du moins en principe, les processus du théâtre même.

Blok est historiquement inséré dans le cadre décrit par Carlson, malgré les efforts d'artistes exceptionnels comme Meyerhold, Artaud, Barba, Brook et Mnouchkine, qui ont, à travers le siècle, cherché à échapper à cette tradition réaliste 5 . Pour cela, ils ont souvent eu recours aux arts traditionnels orientaux, très écartés de la forme du réalisme occidental. Dans les traditions orientales, ces artistes ont découvert un spectacle formel conventionné, codifié. Les frontières entre théâtre, danse et musique n'étaient pas évidentes comme en Occident, et dans le spectaculaire il n'y avait pas de place pour le langage quotidien, réaliste.

Lopposition entre les théâtres de Blok et de Mnouchkine semble évidente, puisque celle-ci rejette le réalisme et le psychologisme 
tant voulu par Blok ${ }^{6}$. Cette catégorisation n'est tout de même pas si simple : Blok nie le théâtral pour rencontrer le "réalisme sain" de Stanislavski, tandis que le rejet de la théâtralité chez Mnouchkine ne se réduit pas à la seule connotation péjorative du terme.

Malgré la ressemblance des termes, ses implications ne sont pas équivalentes : pour Blok, l'antidote à la "théâtralité rigide et stéréotypée" (chez Meyerhold) se retrouverait dans la sincérité et dans le psychologisme des personnages réalistes (chez Stanislavski). Or, la metteuse en scène du Théâtre du Soleil ne veut ni l'un, ni l'autre ! Elle réclame du Théâtre, du Théâtral, du Poétique; et rejette ainsi le réalisme, l'illustratif, le stéréotypé7.

\section{Le Théâtral et le Performatif}

À part les références au théâtre réaliste, certains mots-clés, utilisés par ces artistes, accentuent notre intérêt pour ces deux anecdotes, et peuvent nous servir comme objet d'analyse dans la compréhension générale du terme visé dans cette étude.

Ce sont, entre autres : stéréotype, cliché, et manquelabsence de vérité. À différents niveaux, la notion de théâtralité évoque, tant pour Blok que pour Mnouchkine, le contresens de ce qui fait du théâtre un art du spectacle vivant. Dans cette optique, tout procédé, qu'il soit engagé par l'acteur ou pas, imposant une "scission entre le 'moi' et son 'rôle', suggère inévitablement que ce dernier est moins authentique, plus artificiel" (Carlson, 2012, p. 29). Et c'est précisément en évoquant cette performativité de l'acteur, qu'elle soit authentique ou artificielle, que nous soulèverons notre deuxième problématique : la théâtralité en rapport avec la performativité.

D’après Marvin Carlson (2012, p. 30), dès Sartre, en passant par Artaud, Michael Fried et tout un courant d'artistes et théoriciens modernistes entre les années 1960 et 1980, la théâtralité fut souvent la cible de conceptualisations négatives et limitées, tandis que la performance, au contraire, attirait plus de succès et de visibilité. À propos de cette époque, Carlson reprend David Marshall : "Le type de théâtralité qui paraît suspecte aux yeux de ces théoriciens repose tout particulièrement [...] sur 'son apparence littérale ou figurative de spectacle devant des spectateurs" (Carlson, 2012, p. 30).

D’après Carlson, les attaques écrites contre la théâtralité au long du dernier siècle la lient souvent au vide, au rigide, et au répétitif de 
la représentation, menaçant par son existence l'authenticité du "moi" réel, inédit et subjectif de l'acteur. Depuis les années 1980, avec le boom de popularité qui a gagné le terme performance dans les écrits théoriques, la théâtralité est constamment rejetée, et devient par principe une notion opposée à celle de performativité.

\section{Pour Démystifier la Théâtralité}

Josette Féral intervient alors en proposant sa démystification, par un regard finalement plus positif sur la théâtralité, mais encore loin dans sa complémentarité avec la performance. En 1982, dans une édition spéciale de la revue Modern Drama, elle définissait le théâtre "[...] en tant que structure narrative de représentation qui réinscrivait le sujet dans le symbolique par le biais de 'codes théâtraux', tandis que la performance s'attachait à défaire ces 'codes et compétences', permettant ainsi au 'flux du désir' du sujet de s'exprimer" (Féral, 1982 apud Carlson, 2012, p. 31).

Vingt ans plus tard, dans une autre édition - sous sa propre direction - Féral exprime en note personnelle une correction à la première approche proposée en 1982 : ayant auparavant défini théâtralité et performativité en tant que termes opposés, elle est désormais convaincue que cette opposition est purement rhétorique. Sa redéfinition propose, en bref, que la performativité représente l'aspect singulier et inédit de chaque événement théâtral, tandis que la théâtralité lui permet d'être un processus reconnaissable et significatif pour le spectateur, imprégné de signes, codes et références.

Cette redéfinition se rapproche de celle de Jean Alter (1990), dans l'ouvrage A Sociosemiotic Theory of Theatre publiée en 1990, où la relation entre performance et théâtralité va encore plus loin, comme le suggère Carlson (2012, p. 33):

[le concept de performance] peut être étendu à n'importe quelle composante du théâtre - costumes, décor, éclairage, mise en scène. Ainsi, des costumes éblouissants, des effets de lumière ou des effets scéniques ou encore la façon dont le metteur en scène manipule l'une ou l'autre de ces formes de virtuosité peuvent tous être considérés, d'après Alter, comme une mise en évidence de l'aspect performant, tout comme peut l'être le savoir-faire technique et les qualités artistiques de l'acteur.

Dans cette perspective, la théâtralité ne repose plus sur la forme du spectacle en question, qu'elle soit naturaliste ou stylisée, comme 
le voulaient les mouvements d'avant-garde du début du $\mathrm{XX}^{\mathrm{e}}$ siècle. Elle ne s'oppose plus à la performance, ni ne dépend de la performativité des sujets et des objets impliqués afin de se produire. Elle n'est pas synonyme de rigidité ni d'artificialité, mais d'un discours autonome ayant lieu hors du quotidien, avec ses propres conventions. Cette théâtralité n'est pas une notion qualitative, où il y aurait des sujets plus théâtraux que d'autres. Elle doit, bien entendu, différer au niveau de la réception et décodification des spectateurs, qui peuvent être plus ou moins sensibles/réactifs que d'autres au processus de communication.

Le processus de théâtralisation d'un événement théâtral devient en quelque sorte le résultat d'une suite d'actions performatives de l'artiste et du spectateur, à l'intérieur d'un cadre de conventions théâtrales et culturelles préétablies. Cet accord implicite et sans cesse renouvelé, est précisément ce qui permet au théâtre d'avoir lieu.

\section{Entre Théorie et Pratique}

Les premières réflexions autour de la théâtralité au début du $\mathrm{XX}^{\mathrm{e}}$ siècle sont développées dans le contexte des mouvements d'avantgarde de "rethéâtralisation du théâtre", s'opposant notamment aux traditions du naturalisme et du réalisme. Dans son dictionnaire du théâtre, Pavis (2003, p. 358) définit cette rethéâtralisation:

Alors que le naturalisme efface au maximum les traces de la production théâtrale pour donner l'illusion d'une réalité scénique vraisemblable et naturelle, [...] la rethéâtralisation ne 'cache pas son jeu' et surenchérit sur les règles et les conventions du jeu, présente le spectacle dans sa seule réalité de fiction ludique.

Chez le Russe Nicolas Evreinov, la théâtralité est conçue comme un "instinct de transfiguration" préesthétique, à l'origine de tous les arts et au-delà du théâtre, mais aussi comme une opposition au naturalisme qui produit les "illusions de vie réelle" (Evreinov, 1930, p. 153-154). Plus tard dans les années 1950, Roland Barthes et Bernard Dort, inscrits dans le questionnement du théâtre textocentriste, regardent la théâtralité par la matérialité du corps de l'acteur en dépit du texte: "[la théâtralité] c'est le théâtre moins le texte" (Barthes, 1964, p. 41).

Certaines théories plus contemporaines se limitent à l'analyse sémiologique de la représentation pour en identifier ses signes théâ- 
traux (Fischer-Lichte, 1995), d'autres l'étendent au principe de toute relation de tension entre corps et langage au-delà du théâtre (Bernard, 2012). Toutefois, ce qui représente pour nous l'aspect le plus pertinent des recherches contemporaines, tenant compte de l'interdisciplinarité des arts et de l'imprécision des frontières entre les arts, est le constat suivant : "La théâtralité ne peut plus être envisagée comme une propriété du théâtre mais de tout événement qui, dans un lieu et un temps donnés, rassemble un regardé et un regardant" (Fernandez, 2011, p. 74).

Afin de poursuivre notre étude sur l'émergence de la théâtralité dans le contexte actuel du Théâtre du Soleil, nous fonderons notre analyse notamment sur deux théories contemporaines: celle de Josette Féral (2011), sur les trois clivages de la théâtralité; et celle de Willmar Sauter (2000), sur les trois modes de communication de l'événement théâtral. Introduisons brièvement ces deux théories dans un premier temps, pour ensuite les analyser plus en profondeur.

Insérée dans cette pensée contemporaine déjà mentionnée, Josette Féral a élaboré sa théorie posant la théâtralité sur trois clivages. Le premier se situe entre l'espace du quotidien et celui de la représentation ; le deuxième oppose réel et fiction à l'intérieur de l'espace de représentation; et le troisième s'approche du concept de performativité, concernant l'acteur et la double lecture du spectateur entre ses forces pulsionnelles et le symbolique qu'il représente/interprète.

La théorie de Sauter peut, de façon pertinente, renforcer l'émergence de la théâtralité en parallèle avec les trois clivages de Féral. Il établit un schéma des modes de communication entre performeur (la présentation) et spectateur (la perception), à trois niveaux : le mode sensoriel relève de toute interaction au niveau personnel entre le performeur et son spectateur; le mode artistique représente l'effet esthétique, en termes de genre, style et talent, qui donne un caractère non-quotidien à la communication; et le mode symbolique est fondé sur le caractère représentationnel et fictionnel accepté par le spectateur, en reconnaissant l'altérité de la présentation.

Liviu Dospinescu analyse ces modes de communication à travers les notions contemporaines de performativité et de théâtralité. D'après Dospinescu, la performativité appartiendrait au mode sensoriel, puisqu' étroitement liée à l'interaction émotionnelle et cognitive entre le performeur et le spectateur. Quant à la théâtralité, elle se rappor- 
terait au mode symbolique, définit comme "l'incarnation des actions à des fins de représentation ou de présentation d'images fictionnelles". Le mode artistique, conclut Dospinescu, serait donc "propre tant à la performativité qu'à la théâtralité” (Dospinescu, 2005, p. 201).

L'analyse qui suit sera fondée sur chacun des trois clivages proposés par Féral, et en même temps, traversée par les modes de communication de Sauter. Nous aurons donc l'opportunité d'approfondir ces notions, en rapport avec la pratique observée au Théâtre du Soleil.

\section{La Théâtralité Commence Ailleurs}

Si la théâtralité se produit essentiellement par le regard du spectateur - et si nous considérons que ce spectateur est conscient et informé de l'événement théâtral en question - nous pouvons alors affirmer que cette théâtralité ne se limite pas au moment de la représentation, mais commence bien avant. À ce sujet, André Helbo (2007, p. 63-69) attribue au spectateur le rôle d'actant observateur, ayant la fonction de respecter le contrat spectaculaire propre au théâtre. Dans cette perspective, la sémiologie contemporaine dépasse le principe unidirectionnel d'émission/réception d'un destinateur à son destinataire. Réclamant au spectateur un rôle plus actif dans le processus de communication spectaculaire, la sémiologie nous permet de percevoir l'émergence de la théâtralité en dehors de la relation scène/salle.

Cette notion nous intéresse dans le cas du Théâtre du Soleil, où le lieu et l'accueil du spectateur le préparent en amont à vivre le spectacle. Dans les mots de Mnouchkine, "pour que l'on puisse recevoir le public dans notre maison, dans ce lieu de théâtre, il nous faut créer le monde tous les jours". Ce qu'elle appelle "créer le monde" serait le premier des trois clivages qui constituent la théâtralité : celui qui distingue, à l'égard du spectateur, l'espace quotidien de celui de la représentation.

En achetant son billet et en entrant dans l'espace de représentation, le spectateur signe un accord tacite, un contrat spectaculaire, conférant une signification particulière à tout objet appartenant au monde du spectaculaire : "[...] le spectateur, pour sa part, perçoit les frictions et les tensions entre les différents mondes que la théâtralité met en jeu. Il se trouve contraint, par le fait même, à regarder autre- 


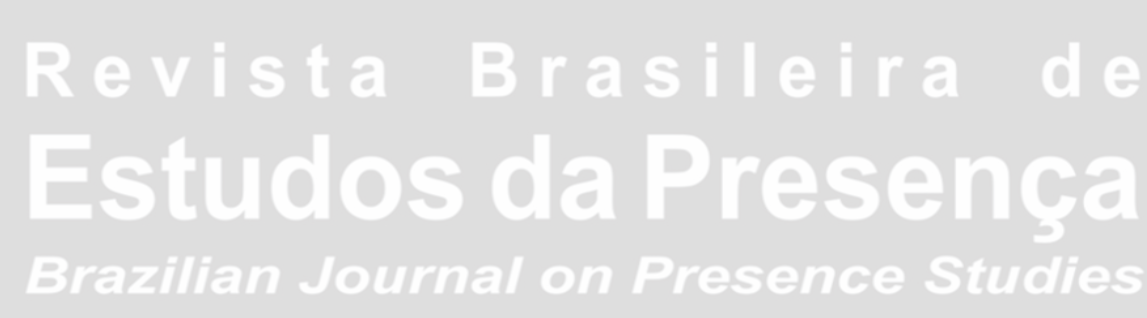

ment" (Féral, 2011, p. 105). Nous sommes ici dans le mode sensoriel proposé par Sauter, où auront lieu les premières interactions avec la présentation spectaculaire, et donc les premières perceptions du spectateur.

Cette notion est plus complexe dans notre objet d'analyse. Soit nous pouvons considérer qu'elle se produit avant de pénétrer dans l'espace de représentation, instaurant une sorte d'entre-deux performatif dans le lieu même du théâtre ; soit nous acceptons que l'espace de représentation puisse s'étendre à d'autres espaces du théâtre, dont le hall d'accueil, la salle à côté des gradins, ou encore dans l'ensemble de la Cartoucherie du Bois de Vincennes - lieu où se trouve le Théâtre du Soleil - dès le franchissement du portail d'entrée.

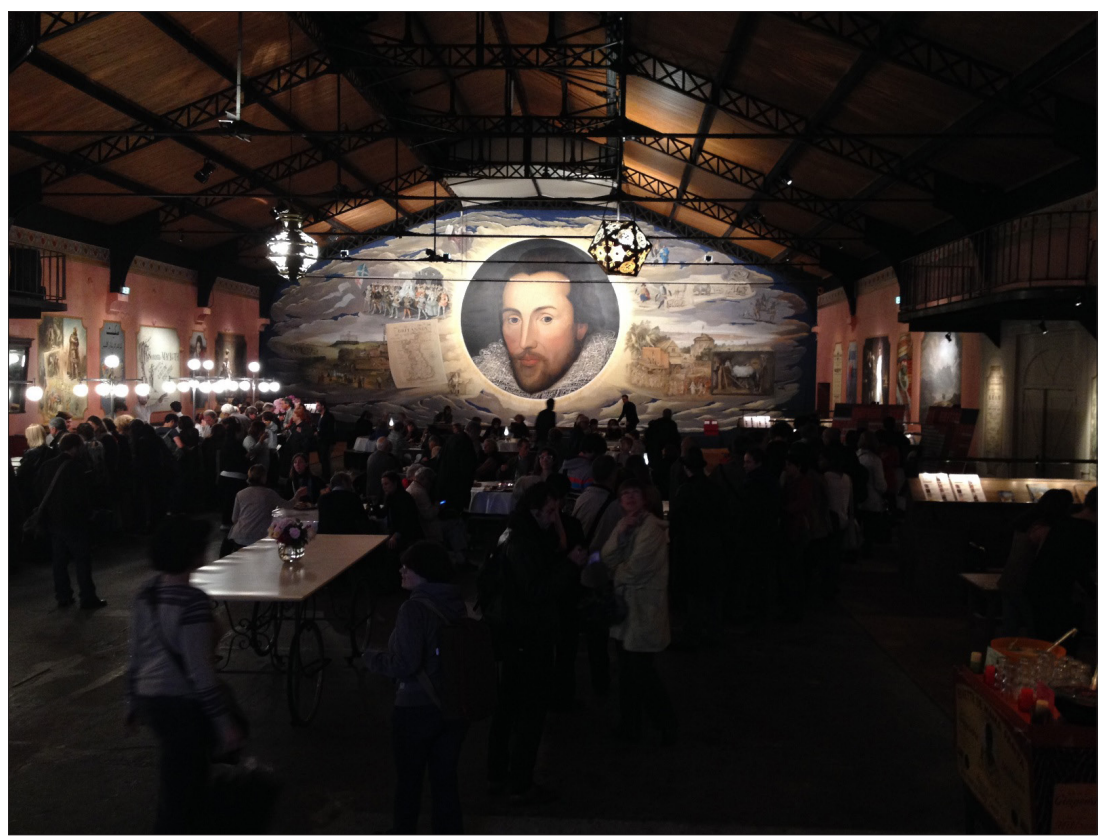

Image 1 - Le public s'installe dans la nef d'accueil du Théâtre du Soleil, une heure avant la représentation de Macbeth. En 2014, à la Cartoucherie de Vincennes, Paris. Photo: Michèle Laurent.

Que nous choisissions l'une ou l'autre de ces approches, le constat de la performativité est évident. Voici l'extrait d'un entretien réalisé avec Ariane Mnouchkine, un mois après la création de Macbeth, en mai 2014:

Stéphane Capron: d'abord, rentrer au Théâtre du Soleil c'est tout un cérémonial. Ariane Mnouchkine vous êtes à l'entrée, vous accueillez les spectateurs, vous déchirez même les billets, et puis le spectacle commence dès l'entrée dans le hall, qui est décoré selon le spectacle. [...] et puis ensuite quand on pénètre dans la salle, sous les gradins, on peut observer les comédiens dans leur intimité. Alors on se fait 
un peu voyeur, on les voit s'habiller, on les voit se maquiller, cela fait partie en fait du spectacle, dès les premières minutes.

Ariane Mnouchkine: au fond, ce qui se passe pendant cette heure, ou ces trois quarts d'heure, pendant qu'ils [les spectateurs] sont avec nous et qu'ils mangent avec nous, c'est aussi qu'ils oublient un petit peu. Ils oublient ce qui s'est passé dans la journée, ils oublient les tensions de la journée, ils oublient le prosaïsme de la vie quotidienne dans une ville comme Paris. Et au fond ils se préparent. Et le hall sert à ça, il sert à montrer le monde tel qu'il pourrait être, c'est-à-dire un lieu où effectivement il y a une convivialité comme on dit, où il y a un respect de l'instant, de l'harmonie, de la qualité des choses que nous tentons de leur offrir (Transcription de l'entretien avec Patrick Cohen, Radio France Inter, le 30 mai 2014).

Comme nous pouvons le noter dans ce bref échange, il existe cette intention, de la part de la troupe, de "préparer le public", de lui permettre de se libérer du quotidien pour plonger dans la poésie du spectacle. Tout geste intentionnel peut être interprété comme un acte performatif, appartenant au rituel théâtral qui provoque ce clivage entre l'espace quotidien et celui de représentation.

Il est important de noter ici que dans l'analyse d'une pratique, nous sommes obligés d'abandonner l'idée de "spectateur idéal ou implicite", comme le souligne Malgorzata Sugiera (2002, p. 226). Évoquons ici l'approche d'André Helbo, qui, d'après l'analyse de Catherine Bouko, propose un spectateur actif et autonome qui "[...] n'est pas seulement confronté à un système de signes qui lui est proposé ; [mais qui] crée véritablement son propre système significatif sur la base de signes émis par l'instance productrice" (Bouko, 2010, p. 219).

D’après Helbo (2007, p. 66):

Tout spectacle implique une multiplicité de pôles, de partenariats, de contextes. Plutôt que la filiation linéaire, c'est le modèle du polysystème qui s'impose, ou mieux encore celui d'une convention labile et qui n'exclut pas la production de texte spectaculaire par le spectateur.

Pourtant, malgré l'infinité et la diversité de textes spectaculaires créés par les spectateurs dans les termes de cet entre-deux, déjà spectaculaire avant la représentation elle-même, la performativité est déjà présente, grâce aux efforts déployés par les artistes. Nous avons retrouvé dans nos notes personnelles de la création de Macbeth un autre exemple du rituel performatif qui suggère ce clivage physique et concret entre les deux mondes: 
Le 29 mai 2014: Le Théâtre du Soleil commémore aujourd'hui les cinquante ans de sa fondation. Comme à l'habitude, vers 18 h 30, une heure avant le spectacle, Ariane a achevé son meeting avec les comédiens et s'est dirigée vers l'entrée du théâtre pour ouvrir les portes au public. Elle réitère le rituel sacré du Théâtre avant d'ouvrir la grande porte rouge, frappe plusieurs fois sur cette porte avant de porter les trois derniers coups, puis ouvre les portes de l'antre magique. Nous étions surpris de voir le public, sans doute sentant qu'il s'agissait d'un jour spécial, l'applaudir longuement devant la porte, avant d'entrer. Quelques-uns étaient très émus et nous le confiaient une fois devant le bar.

Ce geste d'applaudissement des spectateurs à l'extérieur du théâtre, une fois la porte ouverte par Mnouchkine, est une preuve spontanée du clivage effectué simultanément par un ensemble de spectateurs, acceptant d'emblée un mode symbolique de communication. Ceux qui étaient au courant applaudissaient peut-être l'anniversaire des cinquante ans de la troupe, mais en gardant l'altérité (en restant à l'extérieur du théâtre jusqu'à la fin des applaudissements) ils anticipaient - et étendaient - un procédé déjà incrusté dans les conventions culturelles théâtrales entre artiste et spectateur. Cette altérité est indispensable à l'émergence de la théâtralité : elle révèle chez le spectateur la reconnaissance de conventions qui lui permettent de manifester son accord au contrat spectaculaire en question.

\section{Un Va-et-vient entre Réel et Fiction}

Le deuxième clivage proposé par Josette Féral se produit à l'intérieur de l'espace de représentation et concerne encore une fois la distinction que fait le spectateur entre fiction et réalité. Il va et vient entre l'identification des symboles et conventions à lui présentés, son désir de croire à l'illusion, et l'évidence de la vérité matérielle des corps et des objets réels qui l'engendrent. Nous pouvons ici concrètement observer le mode de communication artistique proposé par Willmar Sauter, propre à la performativité et à la théâtralité. Le spectateur commence alors à reconnaitre les conventions qui lui sont présentées, et peut désormais les décoder, selon ses compétences et savoirs, son "encyclopédie personnelle".

Citons Dospinescu (2005, p. 202), qui propose un rapport entre les deux théories: "Le cadre [de théâtralité] est établi par le regard du spectateur ou, du moins, accepté par celui-ci lorsqu'imposé par 
la mise en scène ; le jeu se réfère alors à la valeur symbolique que lui assigne le spectateur à la suite du clivage qu'il opère entre réalité et fiction". Soulignons encore une fois l'existence, au Théâtre du Soleil, de l'intention volontaire des artistes de faire émerger la théâtralité : la transparence, dévoilée par plusieurs procédés, rend évidentes la machinerie du théâtre ainsi que l'émergence de la poésie devant les yeux du spectateur.

L’un des procédés les plus évidents effectués pour ce clivage entre fiction et réalité, est de montrer que l'artiste est en travail ; qu'il se prépare à passer à la fiction. Les loges où les comédiens s'habillent et se maquillent sont situées sous les gradins, et sont visibles par de petites fenêtres incrustées dans un tissu tendu. Bien entendu, les spectateurs ne pénètrent pas dans cet espace, seulement accessible aux regards, et aux flashs des caméras et des téléphones portables. Par ce procédé, le spectateur se sent plus proche de l'intimité des comédiens.

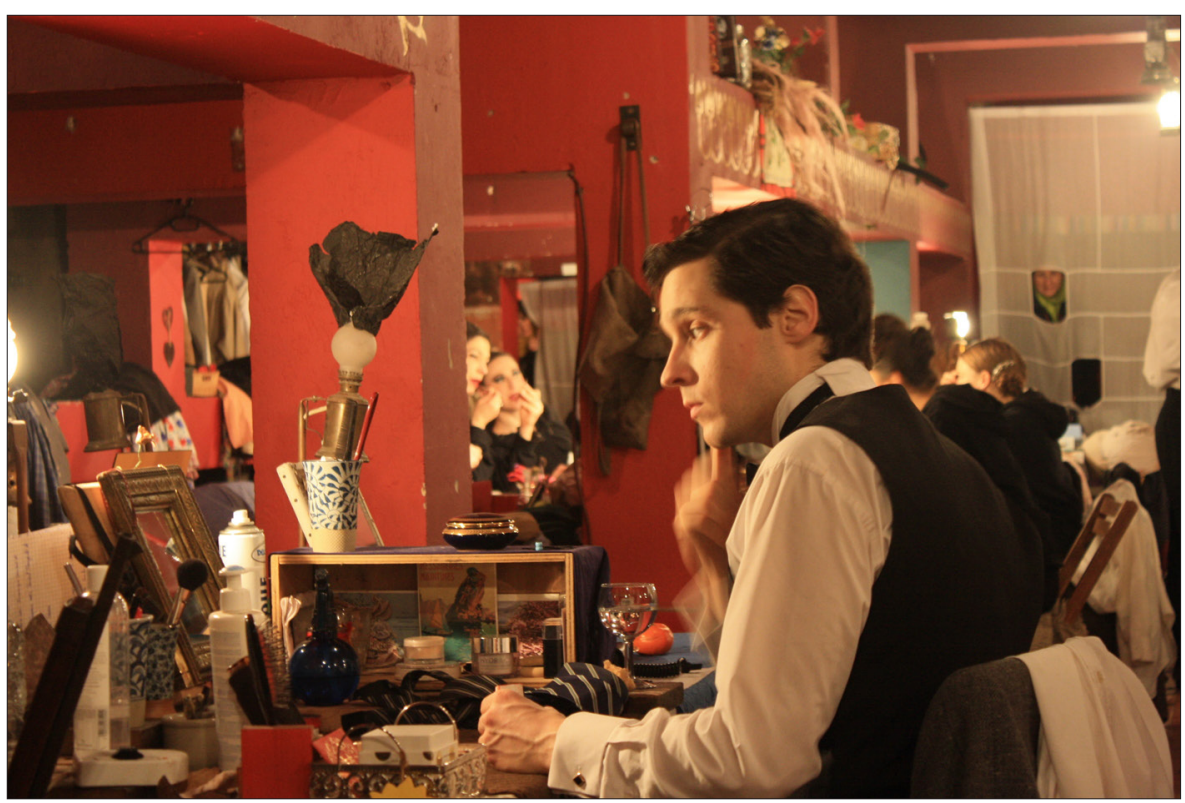

Image 2 - Les loges avant la représentation de Macbeth, d'où on peut voir, au fond, le public qui observe les comédiens. En 2015, à la Cartoucherie de Vincennes, Paris. Photo: Suzana Thomaz.

Cette transparence est ensuite renforcée par la performance du musicien Jean-Jacques Lemêtre: une partie de la musique du spectacle se joue en temps réel sur le plateau, à côté de la scène, où les instruments de musique sont offerts à la vue de tous. Ariane Mnouchkine commente cette notion de transparence ainsi:

Parce que c'est le théâtre! Il ne faut jamais nier le théâtre. La sensation de voir de la vie, de voir plus que de la vie, tout en sachant qu'elle est construite poétiquement ; je pense que c'est ça le comble du plaisir: 
c'est y croire en sachant comment c'est fait. C'est le contraire de l'imposture, le contraire de l'illusion. [...] C'est très très important que la merveille soit possible au fond. C'est-à-dire qu'on sait que cet acteur est en train de mettre son masque, son maquillage, et le public, au fond, est en train d'enlever le sien, pendant ce même moment. On le sent, les gens changent pendant cette heure-là. Ils ont un autre visage, ils se désarment un peu, ils se préparent à recevoir et à faire ce travail qu'on fait au théâtre: on travaille, et le public travaille sans cesse au théâtre (Transcription de l'entretien avec Patrick Cohen, Radio France Inter, le 30 mai 2014).

Il est à noter l'existence de certaines précautions prises avant que "l'intimité" des comédiens ne soit dévoilée au public. Encore une fois, provoqué par cette conscience d'un regardant, le sujet regardé fait son geste performatif. Mnouchkine demande aux comédiens d'être attentifs lorsque "le public rentre chez eux"10 : elle se réfère notamment aux étapes les plus subtiles de la transformation de l'acteur en personnage dans l'habillage et dans le maquillage, où le spectateur, en tant que voyeur, commence en amont à faire ses premières lectures et reconnaissances de la fiction et de ses personnages.

Nous parlons de l'intimité entre guillemets, parce qu'elle doit tout de même être relativisée. Le spectateur ne s'en rend peut-être pas compte, mais cette intimité partagée avec les comédiens est en quelque sorte une performance, un rituel appartenant à l'intégralité de l'événement théâtral. À partir du moment où les comédiens savent qu'ils sont observés, leur comportement change, inévitablement.

Pour garantir leur vraie intimité, dépourvue de tout geste performatif et privée des regards étrangers, il y a, tout près des loges, le gourbi: local où les comédiens gardent leurs affaires personnelles et qui est interdit au public. Malgré la présence du public dans le théâtre, les comédiens partagent leur temps avant le spectacle entre le gourbi et les loges, conscients de ce qu'ils veulent cacher ou montrer aux spectateurs.

\section{Les Forces Symboliques et Pulsionnelles de l'Acteur}

Le troisième et dernier clivage évoqué par Josette Féral concerne le travail de l'acteur, et s'avère donc plus proche du concept de performativité. Il s'agit d'un nouveau va-et-vient, cette fois dans l'équilibre fragile établit par l'acteur entre ses forces symboliques (son altérité, l'illusion suggérée par son jeu, son pas moi) et pulsionnelles (son corps en travail, en performativité, son pas-pas moi). 
Cette approche de Féral intègre, en quelque sorte, les trois modes de communication proposés par Sauter, à différents degrés: la communication sensorielle est encore préservée, tandis que les modes artistique et symbolique se retrouveraient à l'intérieur de ce va-et-vient entre le pulsionnel et le symbolique de l'acteur, dans la mesure où il est perçu par le spectateur, évidemment. À ce sujet, citons Dospinescu (2005, p. 202): "Comme le dit Sauter, en ce qui concerne l'interaction entre présentation et perception, rien n'est symbolique en soi à moins que l'observateur ne le perçoive comme tel'.

Richard Schechner (1985, p. 112) souligne à son tour l'évidence de la théâtralité qui accompagne toujours la performance: le concept de pas moi et pas-pas moi (de l'anglais not me... not not me) fait référence à la double négativité éprouvée par l'acteur de (symboliquement) ne pas être soi-même, puisqu'il joue un autre, tout en restant conscient qu'il l'est sans l'être (pulsionnellement). Josette Féral (2011, p. 90) traduit brièvement ce phénomène, en le liant à la notion de théâtralité : "[...] la théâtralité du performeur se situe donc dans ce déplacement que l'acteur opère entre lui comme soi et lui comme autre, dans cette dynamique qu'il enregistre".

Ne nous éloignons pourtant pas de la place du spectateur dans cette dualité du jeu d'acteur, parce qu'il est, lui aussi, confronté à ce rapport de forces pendant toute la représentation. Nous pouvons d'ailleurs affirmer que ce rapport de forces est encore plus patent chez le spectateur ayant assisté à la préparation des comédiens avant une représentation du Théâtre du Soleil. Cette expérience ne réduit aucunement l'effet d'illusion de la représentation elle-même, mais au contraire, et dans une autre perspective, potentialise la perception du symbolique, accentue les frictions et les tensions de l'action scénique, comme l'explique Josette Féral (2011, p. 105) :

Le regard du spectateur lit donc sur les corps en scène le travail de ces forces toujours à l'œuvre qui relancent sans cesse le processus du jeu et le fragilisent. [...] Il se plaît à reconnaître les signes offerts à son regard et leur subversion permanente par l'acte même d'illusion. Il observe ainsi l'acteur s'efforçant de contrôler cette tension profonde au cœur même du jeu - une tension qui place continuellement ce dernier en danger, en état de vulnérabilité permanente. [...] La beauté du jeu de l'acteur provient précisément de ce combat incessant entre la maîtrise de son corps et les débordements permanents qui le menacent. 


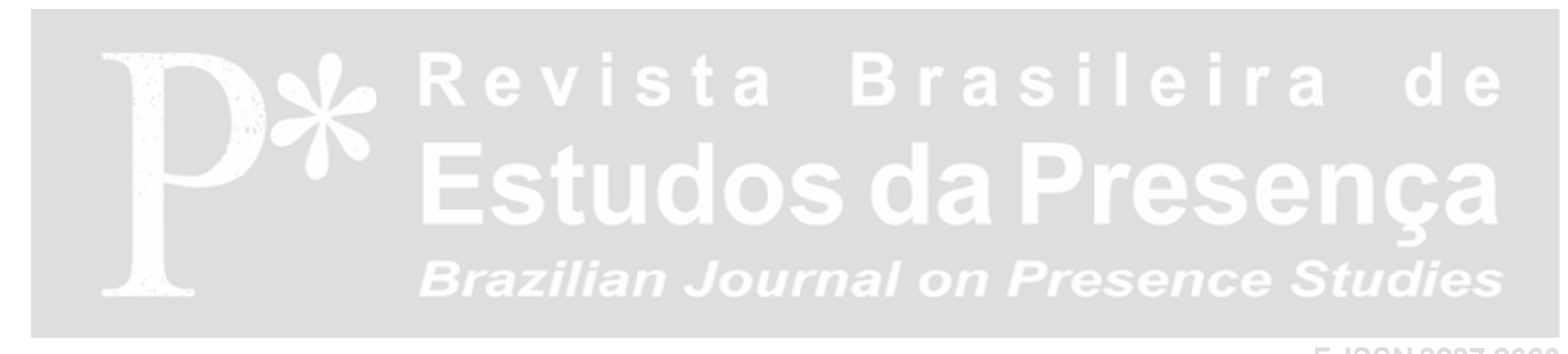

E-ISSN 2237-2660

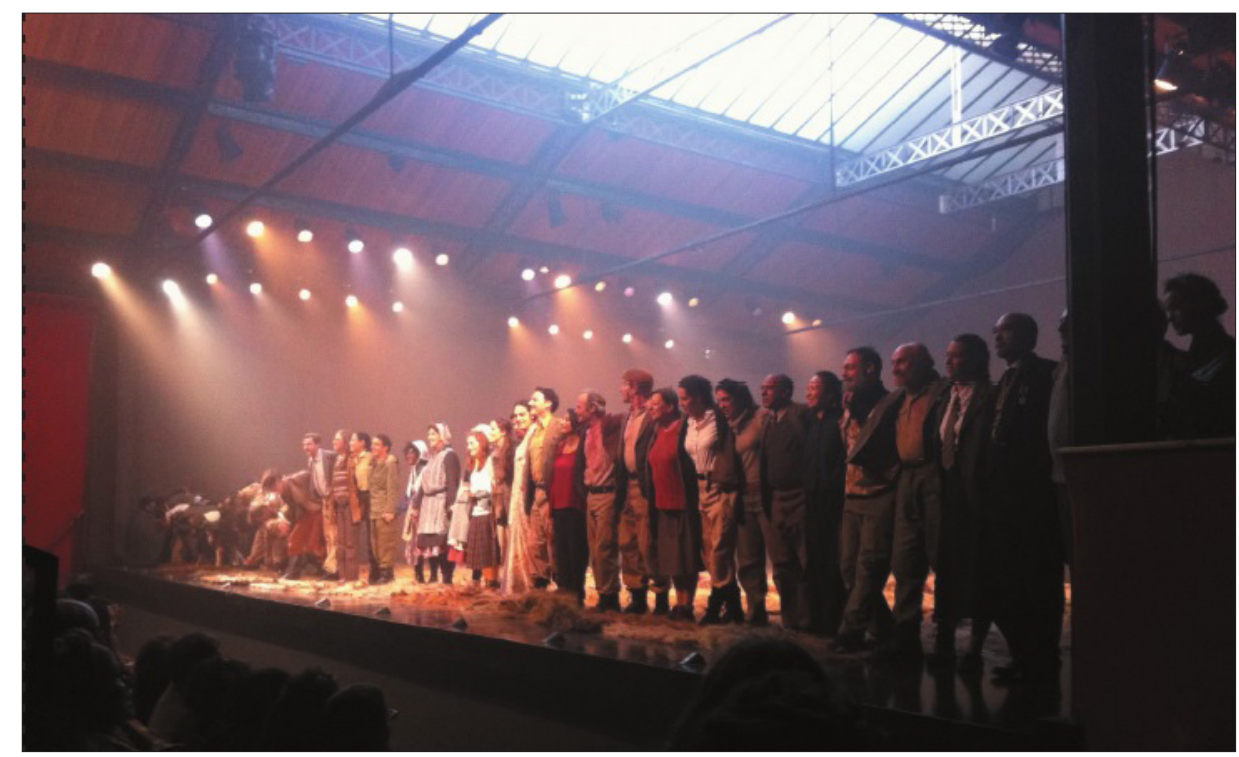

Image 3 - Le salut des comédiens à la fin d'une représentation de Macbeth. En 2014, à la Cartoucherie de Vincennes, Paris. Photo: Charles-Henri Bradier.

La préparation du lieu théâtral, la réception et l'accueil chaleureux des spectateurs, la convivialité suggérée par les espaces communs partagés avec le public dans les heures qui précèdent le spectacle, le contact très proche avec les comédiens au bar ou à travers les fenêtres des loges, et la présence bienveillante de la chef de la troupe, sont des éléments qui servent à potentialiser la qualité du processus de communication instauré entre la troupe du Théâtre du Soleil et son public.

Dans ces conditions, nous croyons avoir suffisamment d'éléments théoriques qui nous permettent d'affirmer une volonté, ou une prédisposition à l'émergence de la théâtralité dans l'événement théâtral présenté par le Théâtre du Soleil dont nous avons pu témoigner pendant la période de création et de représentations de Macbeth, entre octobre 2013 et mars 2015. 


\section{Notes}

${ }^{1}$ Cette réflexion est basée notamment sur l'approche de Josette Féral sur la théâtralité.

${ }^{2}$ Cette phrase est souvent utilisée pour expliquer la dénégation du spectateur de théâtre, brièvement défini ici: "Le mode d'adhésion par identification et dénégation relève du contrat spécifiquement théâtral: le spectateur s'identifie aux personnages tout en étant conscient que l'action scénique est une illusion" (Bouko, 2010, p. 228).

${ }^{3}$ Les deux expressions sont utilisées par Blok: la première décrit le théâtre de Meyerhold, la deuxième celui de Stanislavski (Abensour, 1982, p. 672 et 675).

${ }^{4}$ Cet extrait a été retiré de notre cahier de notes de l'observation participante au sein du Théâtre du Soleil, écrit dans la date qui le précède.

${ }^{5}$ Cet exemple n'est qu'une illustration relevée à partir des deux anecdotes présentées. Le poète Alexandre Blok et tout un mouvement historique ont préféré le réalisme théâtral aux esthétiques proposées par l'avant-garde du début du $\mathrm{XX}^{\mathrm{e}}$ siècle.

${ }^{6}$ La notion de "réalisme” chez Mnouchkine n'est pas précise: pendant les répétitions de Macbeth elle employait le terme pour faire référence au jeu réaliste quotidien, mais aussi pour parler d'un "manque de poésie", de "l'absence du théâtral".

${ }^{7}$ Essayons de préciser cette notion du "théâtral", si valorisée par Ariane Mnouchkine: d'après ce que nous avons pu comprendre en observant sa pratique, le jeu "théâtral" s'équilibre sur un fil menacé par la plupart des conventions occidentales; d'un côté, il risque de tomber dans les ténèbres du réalisme, et de l'autre, dans l'artificiel de la théâtralité.

${ }^{8}$ Ariane Mnouchkine, lors d'une réunion avec les comédiens quelques minutes avant l'entrée du public dans le hall du Théâtre du Soleil.

${ }^{9}$ Terme très répandu dans le domaine de la sémiologie. Citons Helbo (2007, p. 86): “[...] le repérage des traits pertinents dépend de ma connaissance du monde. Dans ce cas, l'iconicité générale (non construite) fait que le signe inclus dans la représentation peut être celui que je connais dans le réel. Un spectacle fondé sur la socialité du comédien [...] n’a de sens que pour celui dont le répertoire culturel permet d'identifier les traits pertinents".

${ }_{10}$ Normalement une demi-heure ou quarante-cinq minutes avant le début du spectacle, l'accès du hall et de la salle de spectacle ainsi que des gradins est ouvert aux spectateurs. Ariane Mnouchkine vient donc prévenir les comédiens en disant "attention, le public rentre chez vous".

\section{Références}

ABENSOUR, Gérard. Blok face à Meyerhold et Stanislavski, ou le problème de la théâtralité. Revue des Études Slaves, Paris, tome 54.4, p. 671-679, 1982.

ALTER, Jean. A Sociosemiotic Theory of Theatre. Philadelphia: University of Pennsylvania, 1990.

BARTHES, Roland. Le Théâtre de Baudelaire (1954). In: BARTHES, Roland. Essais Critiques. Paris: Le Seuil, 1964. P. 41-47. 
BERNARD, Michel. Voix de l'Auteur, Voix de l'Acteur. Théâtre/Public, Paris, n. 205, p. 36-43, automne 2012. (Entre-deux. Du théâtral et du performatif).

BOUKO, Catherine. Théâtre et Réception: le spectateur postdramatique. Bruxelles: Peter Lang, 2010.

CARLSON, Marvin. Résistance à la Théâtralité. Traduit de l'anglais par Virginie Magnat. Théâtre/ Public, Paris, n. 205, p. 28-35, automne 2012. (Entre-deux. Du théâtral et du performatif).

COHEN, Patrick. Radio France Inter. Transcription de l'entretien avec Patrick Cohen le 30 mai 2014. Disponible sur: <http://www.franceinter.fr/player/reecouter?play=909990>. Consulté le 18 juillet 2014.

DOSPINESCU, Liviu. Interfaces et Interférences dans la Communication Théâtrale Moderne: de l'espace vide... et son archétype beckettien. L'Annuaire Théâtral: revue québécoise d'études théâtrales, Montréal, n. 37, p. 197-216, 2005.

EVREINOV, Nicolas. Le Théâtre dans la Vie. Paris: Stock, 1930.

FÉRAL, Josette. Foreword. SubStance: The rise and fall of Theatricality - Special Issue, v. 31, n. 2/3, Issue 98/99, p. 3-13, 2002.

FÉRAL, Josette. Théorie et Pratique du Théâtre: au-delà des limites. Montpellier: L'Entretemps, 2011.

FÉRAL, Josette. Les Paradoxes de la Théâtralité. Théâtre/Public, Paris, n. 205, p. 8-11, automne 2012. (Entre-deux. Du théâtral et du performatif).

FERNANDEZ, Laure. Cadres et Écarts: de la théâtralité en art contemporain, un théâtre à côté du théâtre. In: NAUGRETTE, Catherine. Qu'est-ce que le Contemporain? Paris: L'Harmattan, 2011. P. 73-78. V. 1.

FISCHER-LICHTE, Erika. From Theater to Theatricality: how to construct reality. Theatre Research International, Cambridge, Cambridge University Press, v. 20, n. 2, été 1995.

HELBO, André. Le Théâtre: texte ou spectacle vivant?. Paris: Klincksieck, 2007.

MODERN Drama, Special issue. Theory of Drama and Performance, Toronto: University of Toronto Press, v. 25, n. 1, mars 1982.

PAVIS, Patrice. Dictionnaire du Théâtre. Paris: Armand Colin, 2003.

REINELT, Janelle. La Politique du Discours: performativité et théâtralité. Théâtre/Public, Paris, n. 205, p. 12-20, automne 2012. (Entre-deux. Du théâtral et du performatif).

SAUTER, Willmar. The Theatrical Event: dynamics of performance and reception. Iowa City: University of Iowa Press, 2000.

SCHECHNER, Richard. Between Theater and Anthropology. Philadelphia: University of Pennsylvania Press, 1985.

SUGIERA, Malgorzata. Theatricality and Cognitive Science: The Audience's Perception and Reception. SubStance: The rise and fall of Theatricality - Special Issue, v. 31, n. 2/3, Issue 98/99, p. 225-235, 2002. 
E-ISSN 2237-2660

Suzana Thomaz est comédienne et maître Erasmus Mundus en études théâtrales par l'Université Libre de Bruxelles et l'Université Paris 8. Membre du Théâtre du Soleil depuis 2013, elle approfondit les réflexions autour des fondements esthétiques des processus créatifs de la troupe, portant son regard sur les études culturelles. Suzana prépare à présent une thèse au département théâtre de l'Université Paris 8.

E-mail: suzanacts@gmail.com

Ce texte inédit, révisé par Annelyse Gayraud, est également publié en portugais dans ce numéro.

Reçu le 15 décembre 2014 Accepté le 11 novembre 2015 